\section{Früh erkennen, ob die Krebstherapie anspricht}

Leipzig - Krebs gehört in Deutschland zu den häufigsten Todesursachen. Über den Verlauf einer Krebserkrankung und somit über Leben oder Tod entscheiden häufig eine frühe Diagnostik und die exakte Verlaufskontrolle bei der Therapie. Dabei spielt der kontrastverstärkte Ultraschall (CEUS: Contrast Enhanced Ultrasound) eine immer wichtigere Rolle. Mithilfe dieser strahlenfreien Methode können Ärzte deutlich eher und mit größerer Sicherheit als bei anderen bildgebenden Verfahren erkennen, ob ein Tumor gutoder bösartig ist sowie ob ein Krebspatient gut auf eine Chemo-, Immun- oder Strahlentherapie anspricht. Wie das funktioniert und welches Potenzial in den Ultraschallkontrastmitteln steckt, erläuterten Experten der Deutschen Gesellschaft für Ultraschall in der Medizin e. V. (DEGUM) auf einer Pressekonferenz in Leipzig im Rahmen des Dreiländertreffens der deutschen, österreichischen und Schweizer Ultraschallfachgesellschaften.

Für Patienten ist es eine ganz normale Ultraschalluntersuchung: Mit dem Schallkopf fährt der Arzt den Körper im zu untersuchenden Bereich ab. Der einzige Unterschied: Zu Beginn leitet er wenige Milliliter eines gashaltigen jodfreien Kontrastmittels in die Armvene des Patienten. Die Mikrobläschen von der Größe eines roten Blutkörperchens gelangen in die kleinsten Gefäße und reflektieren die Ultraschallwellen. Bereits wenige Sekunden nach der Injektion machen sie die Organdurchblutung sichtbar. Die inerten Gasbläschen werden nach einigen Minuten über die Lunge wieder abgeatmet und weisen somit im Gegensatz zu anderen Kontrastmitteln (etwa Röntgenkontrastmittel) keinerlei Nebenwirkungen an Schilddrüse oder Niere auf.

„Wir können mithilfe des kontrastverstärkten Ultraschalls bei einigen Tumoren bereits sehr früh vor allem in soliden Organen wie der Leber, der Niere oder der Bauspeicheldrüse erkennen, ob ein Tumor gut- oder bösartig ist“, erklärt Dr. Hans-Peter Weskott. Der Internist ist dieses Jahr Kongress- präsident des Dreiländertreffens der deutschen, österreichischen und Schweizer Ultraschallfachgesellschaften DEGUM, ÖGUM und SGUM in Leipzig. Die Anwendung des kontrastverstärkten Ultraschalls wird beim Kongress Thema in wissenschaftlichen Sitzungen und Modulen sein.

Ein entscheidendes Kriterium für die Beurteilung eines Tumors ist der Grad der Durchblutung. „Dies lässt sich mit dem kontrastverstärkten Ultraschall aufgrund technisch höchster räumlicher Auflösung besonders gut visualisieren und softwareunterstützt quantifizieren“, erläutert Dr. Weskott. Damit kann vielen Patienten eine wiederholte Untersuchung im Computertomografen (CT) oder Magnetresonanztomografen (MRT) erspart werden. Zahlreiche vergleichende Studien belegen eine Gleichwertigkeit des kontrastverstärkten Ultraschalls mit den anderen bildgebenden Verfahren. Für Tumorpatienten, bei denen eine Jodallergie vorliegt, die Nierenfunktion eingeschränkt ist, ein Herzschrittmacher den Einsatz eines MRT verhindert oder eine Strahlenbelastung vermieden werden soll, stellt die Kontrastmittelsonografie das Mittel der Wahl dar.

Auch in der onkologischen Therapie wird die Kontrastmittelsonografie zunehmend angewendet. „So können wir hiermit zu einem sehr frühen Zeitpunkt sehen, ob beispielsweise eine Chemo-, Immun- oder Strahlentherapie erfolgreich anspricht oder ohne Wirkung auf die Tumorentwicklung bleibt, was im CT oft erst viel später sichtbar wird“, erklärt Dr. Weskott. Der Arzt kann mit der CEUS erkennen, ob die Tumordurchblutung dank der Therapie zurückgedrängt oder gar ganz eliminiert werden konnte. „Dies ist entscheidend für einen Krebspatienten, denn wenn wir sehen, dass eine Therapie nicht wirksam ist, muss frühzeitig über Alternativen nachgedacht werden." Auch bei den interventionellen und invasiven Therapieansätzen werden Ultraschallkontrastmittel oft schon beim Eingriff zur Therapiesteuerung und erster Therapiekontrolle eingesetzt. „Bei leberchirurgischen Eingriffen etwa kann die CEUS helfen, bislang unentdeckte kleine Lebertumoren aufzudecken und zum Beispiel mit der Radiofrequenzablation in derselben Sitzung auszuschalten“, so Dr. Weskott weiter. „Mittlerweile ist die CEUS aus dem klinischen Alltag nicht mehr wegzudenken“, betont der DEGUM-Experte - vor allem nicht bei der Behandlung von Krebspatienten.

\section{Literatur}

[1] Sidhu PS, Cantisani V, Dietrich CF et al. The EFSUMB guidelines and recommendations for the clinical practice of contrast-enhanced ultrasound (CEUS) in non-hepatic applications: update 2017 (long version) Ultraschall in Med 2018 Apr; 39: e2-e44

[2] Claudon M, Dietrich CF, Choi BI et al. Guidelines and good clinical practice recommendations for contrast enhanced ultrasound (CEUS) in the liver-update 2012. Ultrasound Med Biol 2013 Feb; 39: 187-210

[3] Schellhaas B, Hammon M, Strobel D et al. Interobserver and intermodality agreement of standardized algorithms for non-invasive diagnosis of hepatocellular carcinoma in high-risk patients: CEUS-LI-RADS versus MRI-LI-RADS. Eur Radiol 2018 Oct; 28 (10): 4254-4264

[4] Zeccolini G, Del Biondo D, Cicero C et al. Comparison of Contrast-Enhanced Ultrasound Scan (CEUS) and MRI in the follow-up of cryoablation for small renal tumors. Experience on 25 cases. Urologia 2014 JanMar; 81 Suppl 23: S1-S8. doi:10.5301/ RU.2014.11986. Epub 2014 Mar 6

[5] Beyer LP, Wassermann F, Pregler B et al. Charakterisierung von fokalen Leberläsionen mit CEUS und MRT mit leberspezifischem Kontrastmittel: Erfahrungen eines radiologischen Zentrums. Ultraschall in Med 2017; 38: 619-625

[6] Kim TK, Noh SY, Wilson SR et al. Contrastenhanced ultrasound (CEUS) liver imaging reporting and data system (LI-RADS) 2017 a review of important differences compared to the CT/MRI system. Clinical and Molecular Hepatology 2017; 23: 280-289 